\title{
El inicial planteamiento zubiriano de la cuestión de Dios en su artículo "En torno al problema de Dios". Influencia heideggeriana y camino propio
}

\section{INTRODUCCIÓN}

Agradezco la invitación a participar en este congreso internacional "La cuestión de Dios en Martin Heidegger. Un desafío para la teología", para dar cuenta del planteamiento "inicial” de Xavier Zubiri sobre la cuestión de Dios, explicitando su dependencia y autonomía respecto del pensamiento del filósofo alemán. Esta oportunidad es particularmente importante porque Zubiri es un pensador que no ha tenido una repercusión significativa en el ambiente teológico chileno, a pesar de contar con una importante obra sobre la temática religiosa (1).

Para dar cuenta de esta opción me limitaré a mostrar el planteamiento zubiriano sobre la cuestión de Dios contenida en su artículo "En torno al problema de Dios" del año 1936. Este texto (2), y una versión ampliada del año 1936, publicada en 1944 en el primer libro del autor titulado Naturaleza, Historia, Dios (3), que corresponde a la que utilizo.

Limitar la presentación del pensamiento zubiriano a su primer artículo sobre el tema de Dios y renunciar explícitamente a una síntesis general, obedece a tres razones que considero necesario explicitar para contextualizar y matizar, en el conjunto de su pensamiento, cuanto aquí indique: en primer lugar, porque este artículo pertenece a los años en que con mayor nitidez se reconoce la gran influencia que la

(1) El grueso de su obra sobre el tema se encuentra recopilada en su trilogía teologal: El hombre y Dios, Madrid 1984 (en adelante HD); El problema filosófico de la historia de las religiones, Madrid 1993 (en adelante PFHR); y El problema teologal del hombre: cristianismo, Madrid 1997 (en adelante PTHC).

(2) Cf. Revista de Occidente 149(1935) 129-159. Reproducida en: Xavier Zubiri, Sobre el problema de la filosofía y otros escritos (1932-1944), Madrid 2002, 215-242 (en adelante SPFOE).

(3) Zubiri retoma este artículo y lo amplía con vistas a una traducción al francés que nunca llega a concretarse por problemas con la calidad de la traducción. Cf. Xavier Zubiri, Naturaleza, Historia, Dios, Madrid 1944 (12 a ed.), 418, nota de Zubiri (en adelante NHD). Para conocer la obra del autor puede verse www.zubiri.net (bibliografía). 
filosofía de Heidegger ha tenido en Zubiri (4); en segundo lugar, porque reconociendo en el autor una evolución en el planteamiento sobre el tema, una progresiva precisión conceptual y cambios temáticos significativos en algunos de sus puntos, entiendo que estructuralmente su planteamiento inicial se mantiene vigente en el tiempo (5); y, finalmente, porque me parece muy difícil realizar una adecuada presentación de su pensamiento general sobre el problema de Dios o teologal en un lapso tan limitado.

El artículo "En torno al problema de Dios" es particularmente importante para conocer el planteamiento zubiriano, debido a que es la primera vez que utiliza el concepto de "religación". Si bien este no es la única clave de lectura posible del tema en el autor, sí es preciso reconocer que se trata, sin lugar a dudas, de un concepto clave al que dedica una permanente atención en el tiempo (6).

Para comprender el planteamiento zubiriano sobre este problema, es necesario considerar su aporte en continuidad con el horizonte de la tradición que lo antecede, lo acoge, lo forma y también le demanda una respuesta adecuada a los desafíos de su tiempo. Solo así será posible reconocer y valorar razonablemente su esfuerzo vital por repensar filosóficamente el problema de Dios buscando una mayor radicalidad (7).

En función del objetivo propuesto divido el trabajo en dos puntos: en el primero, presento a grandes rasgos la influencia heideggeriana en Zubiri y su continuidad discontinua y, en el segundo, expongo sintéticamente el planteamiento zubiriano presente en su artículo "En torno al problema de Dios". Para finalizar la exposición con una breve conclusión.

\section{INFLUENCIA HEIDEGGERIANA (8)}

El pensamiento de Zubiri debe comprenderse en "continuidad" respecto del horizonte abierto por la fenomenología de Husserl, que posibilita un espacio de

(4) Además de la influencia heideggeriana se reconoce en este texto la presencia de: la metafísica griega, NHD 436-440 y 442-443; de Boecio y de san Agustín, NHD 425; de santo Tomás y el tomismo, NHD 443-444; san Buenaventura, NHD 431; de la mística especulativa, NHD 441-442; de la filosofía moderna, NHD 425-426; de Blondel, NHD 434; Ortega, NHD 452. n. 2; y otros.

(5) Cf. HD.

(6) Para profundizar en el planteamiento de Zubiri sobre la religación ver HD 15-112. En esta primera parte, que data de 1983, se encuentra la exposición más madura de Zubiri sobre el tema; PFHR 13-77.

(7) Cf. Jordi Corominas y Joan Albert Vicens, Xavier Zubiri. La soledad sonora, Barcelona 2006 (en adelante J. Corominas, Xavier Zubiri). Este texto presenta una importante contextualización de la vida y obra de Zubiri en la historia social, política, religiosa e intelectual de España y Europa. Ello permite comprender el desarrollo religioso intelectual del autor, que indudablemente esta condicionado por su experiencia vital.

(8) Cf. J. Corominas, Xavier Zubiri, 217-223; Enzo Solari, La raíz de lo sagrado. Contribuciones de Zubiri a la filosofía de la religión. Tesis doctoral presentada a Hochschule für Philosophie/ Philosophische Fakultät S.J., München 2007, 76-98 (en adelante E. Solari, La raíz); Diego Gracia, Voluntad de verdad, Barcelona 1986, 23-74 (en adelante D. Gracia, Voluntad); Diego Gracia, "Zubiri y la filosofía de la religión (1934-1944)", en: The Xavier Zubiri Review, Vol. 8, 2006, 59-92 (en adelante D. Gracia, "Zubiri y la filosofía”). 
libertad para el quehacer filosófico frente a la gran influencia de la filosofía idealista, positivista, psicologista y neokantiana. Es decir, básicamente en continuidad con los proyectos fenomenológicos de Husserl, Ortega y Heidegger.

Reconociendo esta continuidad, es preciso "distinguir" la propuesta zubiriana de los proyectos fenomenológicos de estos autores, ya que es el propio Zubiri quien indica, en el prólogo a la edición inglesa de NHD de 1980 (9), que su filosofía madura no es fenomenología ni ontología, sino filosofía de lo real en cuanto real, es decir, metafísica (10). En este mismo texto indica que el desarrollo de su actividad filosófica está marcado fundamentalmente por tres etapas: la primera, que llega aproximadamente hasta 1930, puede ser entendida como "fenomenológica" (11) y, en gran medida, está influenciada por Husserl y Ortega, su maestro en España; la segunda, dominada por la influencia del pensamiento de Heidegger, puede ser caracterizada como "ontológica" y se prolonga aproximadamente hasta 1944. Desde esta fecha comenzaría, según su propio testimonio, una nueva etapa que considera de maduración y que denomina explícitamente como "metafísica". Esta última corresponde al desarrollo más original de su pensamiento y gira en torno a las ideas de "realidad" e "inteligencia sentiente" (12).

Zubiri conoce algunos escritos de Heidegger, especialmente Ser y tiempo, Qué es metafísica, texto que además traduce, El origen de la obra de arte y la Carta sobre el Humanismo, dando cuenta de este pensamiento en algunas de sus exposiciones y obras (13). Pero también tuvo acceso a los apuntes de los cursos que el filósofo alemán impartió entre 1920-1930 y asistió a sus cursos en Freiburgo, entre los años 1928 y 1929. El planteamiento heideggeriano de estos años impresionó realmente a Zubiri por su profundidad y novedad, y porque entendió que sus propias ideas estaban reflejadas en él. De esta gran sintonía da cuenta una reciente biografía del autor:

"La publicación de abril de 1927 del libro de Heidegger Ser y tiempo, una obra que va a ser decisiva en el panorama filosófico del siglo $\mathrm{XX}$, convierte este interés en algo acuciante. Zubiri se da cuenta inmediatamente del papel crucial que va a desempeñar este trabajo en su propio destino filosófico y se dedica todo el año a leerlo y releerlo: 'Sin saber por qué, el espíritu del libro y muchos de sus

(9) Cf. NHD 9-16.

(10) Cf. NHD 16.

(11) A pesar de lo indicado por el propio Zubiri en este prólogo, creo que finalmente nunca abandona el ideal del método fenomenológico. Cf. Xavier Zubiri, Sobre la esencia, Madrid 1962 (en adelante SE) y su trilogía IS (Inteligencia Sentiente), nombre que reservo para la trilogía completa compuesta por: Inteligencia y realidad, Madrid 1980 (en adelante IRE); Inteligencia y logos, Madrid 1982 (en adelante IL); Inteligencia y razón, Madrid 1983 (en adelante IRA).

(12) Zubiri expone su idea de realidad e inteligencia respectivamente en sus obras de madurez: SE e IS. Para conocer más detalles de estas periodificaciones ver Antonio Pintor Ramos, Génesis y formación de la filosofía de Zubiri, Salamanca 1994, 31-56- (en adelante Pintor Ramos, Génesis); D. Gracia, Voluntad, 19-30. Para profundizar en la continuidad y originalidad del pensamiento maduro de Zubiri ver Jorge Eduardo Rivera, Heidegger y Zubiri, Santiago 2001 (en adelante J. E. Rivera, Heidegger).

(13) Cf. SE, 438-453; Xavier Zubiri, Cinco lecciones de Filosofía, Madrid 1988, 261-271 (en adelante CLF); Xavier Zubiri, Sobre el sentimiento y la volición, Madrid 1992, 325 (en adelante SSV); y PFHR, 24-27. 
pensamientos me parecieron extraordinariamente naturales. Quedé sorprendido. Comprendí entonces que cuando uno se sorprende de una naturalidad empieza a comprender'. Le parece que alguien ha conseguido por fin poner por escrito ideas que a él hace tiempo que le bullen en la mente" (14).

Zubiri reconoce los aportes de la filosofía heideggeriana en diversos temas y es notoria su influencia en los trabajos que realiza entre los años 1930-1944, algunos de los cuales fueron reunidos y publicados en NHD (15). Como ejemplo de esta influencia puede indicarse: la analítica de la existencia (16); la necesaria diferenciación entre ente y ser (17); la idea de descubrimiento o verdad (18); de temporalidad (19); la afirmación de que el hombre está inmerso, implantado inquieta y transcendentalmente en el ser (20); la comprensión de la idea del ser y el llamado de atención ante su aparente carencia de supuestos (21), etc. Además, Zubiri comparte con Heidegger la necesaria óptica histórica y el anhelo fenomenológico de una filosofía pura y libre de los presupuestos teológicos que la han condicionado históricamente en occidente. Reflexiona sobre la función filosófica que ha ejercido la teología y sobre las funciones teológicas de la metafísica occidental. Comparte el recuento histórico de la filosofía europea a la luz de su carácter metafísico y ontoteo-lógico, llegando a formular su idea de los dos horizontes de la filosofía de occidente (22).

Pero la relación entre ambos pensadores no es solo de continuidad sino también de distinción. Con el tiempo y a pesar de su gran admiración por el planteamiento heideggeriano, será inevitable el conflicto con su ontología mundana e histórica (23). Conflicto que se profundiza a partir de 1944 y que es testimoniado por una serie de ejemplos en sus cursos. Zubiri discute fundamentalmente las tesis centrales de la época de Ser y tiempo, afirmando que la existencia no es una apertura que reposa sobre sí misma ni es un puro evento histórico (24); que la idea del ser no debe sustantivarse (25); que el ser se funda en el tiempo y no al revés (26); y llama

(14) J. Corominas, Xavier Zubiri, 190. La cita es de una carta de Zubiri a Heidegger fechada el 19-II1930 .

(15) Las últimas publicaciones póstumas han incluido los documentos de este período que no fueron incorporados por Zubiri en su primer libro.

(16) Cf. NHD 423-426.

(17) Cf. NHD 13-19.

(18) Cf. SPFOE 23-24, n. 4

(19) Cf. SPFOE XII-XIII. El editor da cuenta de la intención de Zubiri de escribir un libro "Sobre el problema de la filosofía", en que el tema de la temporalidad jugaría un rol central como nuevo horizonte de la filosofía. Obviamente en esto Zubiri está fuertemente influenciado por las ideas de Ser y tiempo.

(20) Cf. SPFOE 299.

(21) Cf. NHD 387-388. Para una detallada exposición de la influencia heideggeriana de estos años ver Solari, La raíz, 94-95.

(22) Cf. SPFOE 17-125

(23) Cf. NHD 14-15.

(24) Xavier Zubiri, Estructura dinámica de la realidad, Madrid 1989, 102 (en adelante EDR); Cf. SE 4-5.

(25) Cf. NHD 16.

(26) Cf. EDR 296, 306. 
la atención sobre una lectura arbitraria de la historia de la filosofía por parte de Heidegger (27).

En estas discrepancias ya es posible reconocer, aunque sea germinalmente, algunas de las ideas propias de la filosofía madura de Zubiri. Un ejemplo de esto es su afirmación de que en la proclamación del ser existe un olvido de la realidad primaria de las cosas (28) y de que la verdad como desvelación se funda en una verdad real que es anterior (29). Pero las discrepancias definitivas aparecerán finalmente en sus obras de madurez, es decir, en Sobre la esencia e Inteligencia sentiente en que discute las ideas heideggerianas de sentido, comprensión, desvelación, ser e historicidad.

\section{2. “EN TORNO AL PROBLEMA DE DIOS” (30)}

Zubiri estructura este texto, además de la introducción y la conclusión, en cinco puntos en los que desarrolla el cuerpo del trabajo. En estos aborda respectivamente los temas de: la "existencia y religación: el problema de Dios"; los "errores de comprensión en el planteamiento del problema"; el "haber y ser: Dios y el problema del ser"; la "Religación y libertad"; y finalmente, "el problema del ateísmo: la soberbia de la vida". En esta oportunidad me limitaré a presentar los elementos más significativos de los cuatro primeros puntos, dejando para otro momento el tema del ateísmo, respecto del cual solo quisiera indicar que con el tiempo existe un cambio fundamental en la comprensión zubiriana (31).

El artículo comienza reconociendo la ambigüedad de la expresión "problema de Dios" y aclarando el sentido en el que será abordado el tema. Indica expresamente que no tratará de Dios en sí mismo, sino de la posibilidad filosófica del "problema" de Dios, que no es ni la cuestión de su existencia ni la del concepto de Dios, sino solamente la de "la constitutiva y ontológica religación de la existencia" (32). Zubiri no busca probar ni negar racionalmente la existencia de Dios, ni determinar el órgano o facultad más adecuada para acceder a Él (33), sino simplemente explicitar el "supuesto" que está a la base de toda demostración, negación o sentimiento respecto de su existencia.

Hecha esta aclaración fundamental, intenta contextualizar la cuestión de Dios estableciendo una analogía con el problema filosófico de la existencia de un mundo “exterior". Sobre este aspecto, valora el replanteamiento realizado por la fenomenología contemporánea y repasa brevemente las actitudes anteriores ante la cuestión: la "idealista", que negaba la existencia de cosas reales externas e independientes del sujeto, y la posibilidad de conocerlas; y la "realista", en su doble vertiente de

(27) Cf. Xavier Zubiri, Los problemas filosóficos de la metafísica occidental, Madrid 1994, 39-40 y 90-91 (en adelante PFMO).

(28) Cf. Xavier Zubiri, Sobre el hombre, Madrid 1986, 676 (en adelante SH).

(29) Cf. PFMO 339.

(30) Cf. NHD 417-454.

(31) Cf. HD 11-12, 164, 260-261, 267, 281-287, 294, 342-343, 370, 376.

(32) NHD 454.

(33) Cf. NHD 420, 423. 
realismo "crítico" o "ingenuo", que admitiendo la existencia del mundo exterior y del sujeto, afirmaban respectivamente su existencia como fruto de un razonamiento fundado en un hecho evidente o en la fuerza de la evidencia de un hecho externo al hombre.

A su juicio, tanto el idealismo como el realismo tienen un mismo supuesto ya que ambos parten de la consideración de la existencia o inexistencia del mundo exterior como si se tratara de un hecho "extrínseco" y "añadido" a la existencia del sujeto. Un supuesto que ha sido radicalmente cuestionado por la fenomenología que comprende al sujeto humano como "constitutiva" y "formalmente" abierto a las cosas, posibilitando la comprensión de la "exterioridad" del mundo como una dimensión de la propia estructura ontológica formal del hombre, no como un simple "hecho" (34), y la "existencia de un mundo exterior" como algo que le viene al hombre desde sí mismo, no como algo que proviene de fuera (35). En este sentido, Zubiri reconoce y valora el aporte de la fenomenología que permitió reformular adecuadamente el problema de la realidad del mundo exterior, superando tanto el idealismo como los realismos, al comprender que el mundo exterior ya no puede ser pensado como un "hecho" extrínseco y añadido al sujeto, sino como una dimensión constitutivo-formal del propio ser humano.

Guardando las proporciones, afirma que el problema de Dios no ha corrido la misma suerte. Por el contrario, cada vez que la filosofía aborda este tema lo hace partiendo del supuesto "acrítico" de que el hombre y las cosas son sustantes o sustantivas y que, por lo tanto, en caso de que existiera Dios necesariamente sería extrínseco a las cosas sustantes. Si a esto se suma la afirmación de la incapacidad de la razón humana de conocer a Dios, sin explicar mayormente en qué consiste esta limitación, queda abierta la puerta para que quienes no admiten la posibilidad de este conocimiento vean en esto la entrada al sentimiento y a lo irracional, y centren la discusión en la importancia del órgano para acceder a Dios, olvidando el tema de fondo que es el problema de Dios en sí mismo.

Ante el descentramiento del verdadero problema, Zubiri plantea un cuestionamiento fundamental a los supuestos aceptados comúnmente en relación con el tema. Cuestiona el que la existencia de Dios sea entendida tan solo como un hecho "exterior" al hombre; si el acceso a Dios es consecutivo o constitutivo al modo de ser de la razón; si entrar en relación con Dios depende de un órgano o facultad, sean estos los sentimientos o la razón; o si, más bien, el ser del hombre es constitutivamente un ser "en" Dios (36).

Lo importante es comprender que ya en la introducción, aunque sin nombrarla expresamente, Zubiri afirma que el problema de Dios tiene por raíz la religación de la existencia (37), como algo que se halla en la propia existencia humana, y no fuera o más allá de ella y del mundo. El problema de Dios es una dimensión radical de la vida humana, a tal punto que puede indicarse que el hombre consiste en el problema

(34) Cf. NHD 421.

(35) Cf. NHD 421-422.

(36) Cf. NHD 423.

(37) Es interesante constatar que en esta etapa inicial Zubiri todavía no distingue entre Dios y religación, aspecto que irá aclarando hasta llegar al planteamiento de HD. 
mismo de Dios. Solo de esta manera se entiende su afirmación de que la cuestión acerca de Dios se retrotrae finalmente a una cuestión acerca del hombre (38). Es decir, que el problema filosófico de Dios consiste finalmente "en descubrir la dimensión humana dentro de la cual esa cuestión ha de plantearse, mejor dicho, está ya planteada" (39). Para Zubiri, el ser humano "es" el problema de Dios porque es una existencia constitutivamente religada.

\subsection{Existencia y religación}

Una vez situado el problema de Dios en íntima relación con el hombre, Zubiri realiza una exposición de los rasgos existenciales que demandan la religación. En este punto se puede reconocer que sigue muy de cerca el planteamiento heideggeriano y de ello dan buena cuenta las siguientes palabras del propio autor:

"La existencia humana, se nos dice hoy, es una realidad que consiste en encontrarse entre las cosas y hacerse a sí misma, cuidándose de ellas y arrastrada por ellas (...) La existencia humana está arrojada entre las cosas, y en este arrojamiento cobra ella el arrojo de existir. La constitutiva indigencia del hombre, ese su no ser nada sin su ser con y por las cosas, es consecuencia de este estar arrojado, de esta su nihilidad ontológica radical” (40).

Zubiri ha estudiado detenidamente los planteamientos filosóficos de su tiempo y conoce la centralidad que en estos ha adquirido la existencia humana, como una realidad que se "hace" a sí misma "entre" las cosas en que se encuentra. Comprende que es en este sentido en que se habla de una existencia "arrojada" entre las cosas que, en el mismo movimiento de estar arrojada, cobra el "arrojo" de existir. Condición que es, al mismo tiempo, la constitutiva indigencia y la máxima nihilidad ontológica del hombre, ya que este no es nada si no es "con" y "por" las cosas.

Pero aun reconociendo que se mantiene en el horizonte de la analítica de la existencia heideggeriana, en este texto ya se aprecian algunos elementos que señalan el progresivo camino de distanciamiento respecto de las posturas de Heidegger. Zubiri valora este aporte pero cree que es posible una mayor radicalidad en el planteamiento de la existencia humana. En función de esta percepción, se pregunta por la relación del hombre con la totalidad de su existencia y por el carácter mismo del "estar arrojado" entre las cosas, cuestionándose si este no es más bien el "encontrarse" existiendo que el "existir" mismo. Ello haría más honda y radical la constitutiva nihilidad ontológica del hombre de lo que se ha afirmado hasta el momento.

Por eso se esforzará en mostrar la falta de precisión con que está descrita la "existencia" al no acentuar suficientemente que el hombre se "encuentra" existiendo. Considera, por tanto, insuficiente concluir que el hombre se encuentra "existiendo", porque con ello puede dejarse en segundo plano el fundamental y primario

(38) Cf. NHD 423.

(39) NHD 423.

(40) NHD 423-424. 
"encontrarse" (41). Zubiri entiende que existir puede significar la "manera de ser" del hombre, es decir, la manera de transcender y de vivir. Pero también puede significar "el ser mismo" del hombre, aquello que es conquistado justamente al vivir y al transcender. Por eso afirma que existir es vivir para ser, en una clara orientación hacia la idea de persona y no de naturaleza, es decir, en la línea de aquello "que" se es y no de aquello "por" lo que se es (42). La persona es el ser del hombre, el ser en el que se encuentra implantado y en que se realiza (43).

"la persona es el ser del hombre. La persona se encuentra implantada en el ser 'para' realizarse. Esa unidad, radical e incomunicable, que es la persona, se realiza a sí misma mediante la complejidad del vivir. Y vivir es vivir con las cosas, con los demás y nosotros mismos, en cuanto vivientes. Este 'con' no es una simple yuxtaposición de la persona y de la vida: el 'con' es uno de los caracteres ontológicos formales de la persona humana en cuanto tal, y, en su virtud, la vida de todo ser humano es, constitutivamente, "personal'" (44).

Zubiri indica que el hombre se encuentra "implantado" y no "arrojado" en el "ser" y señala que prefiere el concepto "ser" al de "existencia" debido a la ambigüedad de esta última expresión, que no distingue adecuadamente entre naturaleza y persona (45). Por lo tanto, la existencia ya no es un puro arrojamiento ni implante en el ser sino una auténtica religación.

“(...) preferiría decir que el hombre se encuentra, en algún modo, implantado en la existencia (...), que el hombre se encuentra implantado en el ser" (46).

En un intento por una mayor radicalidad, Zubiri sostiene once tesis que señalan que el hombre es una existencia radicalmente religada (47). Aunque en algunas de estas tesis se mantiene en el pensamiento heideggeriano ya es posible encontrar el inicio de un camino distinto (48).

$1^{\text {a }}$ tesis:

Afirma que "El hombre existe ya como persona" (49). Siguiendo a Heidegger afirma que el hombre existe pero agrega que existe "ya" como persona. El hombre es un ente cuyo ser consiste en tener que realizarse como persona en la vida. Y

(41) Cf. NHD 424. Sobre este punto Zubiri nuevamente señala la utilidad de la analogía del mundo exterior al que hacía referencia en la introducción del artículo.

(42) Cf. NHD 425-426. Zubiri sigue la idea de "persona" de Boecio, San Agustín, Descartes, Locke y Kant.

(43) Zubiri parece reconocer en esta formulación una insuficiencia que explicaría su futura postura respecto de la realidad y del ser.

(44) NHD 426.

(45) Cf. NHD 424-425

(46) NHD 424.

(47) Cf. Diego Gracia, "Zubiri y la experiencia teologal. La difícil tarea de pensar a Dios y la religión a la altura del sigo XX", en: P. Brickle (ed.), La filosofía como pasión, Madrid 2003, 251-263.

(48) Cf. E. Solari, La raíz, 139-147.

(49) NHD 427. 
sostiene, en un planteamiento similar al de Ser y tiempo, que el hombre tiene que realizarse y elaborar la personalidad.

$2^{a}$ tesis:

Afirma que "El hombre se encuentra enviado a la existencia, o mejor dicho, la existencia le está enviada" (50). El carácter misivo de la existencia señala que la facticidad de la vida no es puesta por el mismo hombre sino que le está dada al hombre. Este carácter misivo es propio de la vida, en el sentido de que la vida misma "es" misión y no solamente "tiene" una misión. La vida ya no es un simple factum, ni la existencia una espléndida posibilidad, sino algo más:

"El hombre recibe la existencia como algo impuesto a él. El hombre está atado a la vida. Pero, como veremos más tarde, atado $a$ la vida no significa atado por la vida" (51).

Es importante constatar que en una nota manuscrita, el propio autor, indica que atado "a" la vida no significa atado "por" la vida sino por "la realidad". Una afirmación que no aparece en el texto de 1935 y que da cuenta de un proceso de maduración posterior.

Si bien, en este punto, puede hablarse de una posición común a ambos autores ya comienzan a distinguirse sus caminos, en la medida en que es preciso atender al carácter impuesto de la existencia que se padece y soporta por algo que hace que haya que soportarla. En este mismo sentido se expresa Solari al afirmar que:

"Ese tener que ser (Zusein) podría ser identificado con esta idea del envío, de la misión existencial. Pero hemos de atender a ese carácter según el cual el ser del hombre está "atado a la vida". Tal atadura no es un puro factum ("la presunta facticidad de la existencia es solo una denominación provisional"), ni es tampoco una posibilidad, por excelente que esta sea ("una espléndida posibilidad"). Facticidad y posibilidad son las denominaciones que otorga Heidegger al existir en tanto que envío. Zubiri va más allá. La vida es una misión que, en un sentido preciso y no meramente metafórico, se soporta y padece: "el hombre recibe la existencia como algo impuesto a él". Y se la soporta y padece no por sí misma sino por algo que hace que haya que soportarla y padecerla: "atado $a$ la vida no significa atado por la vida" (52).

$3^{\mathrm{a}}$ tesis:

Es la existencia la que le "impone" al hombre lo que "le hace" vivir y no las cosas. Estas le entregan estímulos y posibilidades "para" vivir pero no lo "hacen" vivir. Lo que le impone la existencia y lo impulsa a vivir es el impulso para ser. Por eso, para Zubiri la vida es más que estímulos y posibilidades, es una realidad que está impulsada y que tiene que realizarse en cuanto tal.

(50) NHD 427.

(51) NHD 427.

(52) Solari, La raíz, 139. 
$4^{\mathrm{a}}$ tesis:

Lo que hace vivir al hombre no es un apego o tendencia natural a la vida, como plantean algunas filosofías vitalistas o comprensiones biológicas, sino "algo anterior" en que el hombre se apoya para existir y hacerse. El hombre no solo se encuentra con las cosas, con las que tiene que hacer su ser, sino que se encuentra apoyado en algo a tergo, es decir, con una fuerza ejercida desde atrás que lo impulsa, de donde le viene la vida misma. Esto que apoya al hombre es justamente lo que le impone la vida y lo impulsa a vivir.

$5^{\text {a }}$ tesis:

Pero este "algo" anterior, que apoya al hombre en la existencia y lo hace ser, no es algo físico sino una "fuerza" (53). Al hombre no le basta con poder y tener que hacerse, necesita la fuerza de estar haciéndose, necesita que "le hagan hacerse" a sí mismo, porque su nihilidad es tan radical que necesita de un punto de apoyo desde el cual puede y tiene que hacerse a sí mismo, ya que por sí solo no tiene la fuerza para realizarse. En este sentido, la nihilidad zubiriana es distinta a la heideggeriana, ya que no basta con afirmar que el hombre "tiene" y "puede" hacerse, sino que es preciso reconocer que requiere que le "hagan" hacerse. Zubiri entiende que la existencia es un hecho radical pero un hecho "radicalmente radicado", que obliga al hombre a preguntarse por la fuerza en que la existencia está apoyada.

"El hombre no solo no es nada sin cosas, sino que, por sí mismo, no "es". No le basta poder y tener que hacerse. Necesita la fuerza de estar haciéndose. Necesita que le hagan hacerse a sí mismo" (54).

$6^{\mathrm{a}}$ tesis:

Esta fuerza que hace al hombre ser, sin ser él mismo la fuerza, es paradójicamente al mismo tiempo, lo más propio de sí, porque le hace "ser", y lo más otro de sí, porque le "hace" ser.

$7^{\text {a }}$ tesis:

El hombre, al existir, se encuentra con que hay cosas, hay que hacerse y debe estar haciéndose, y en ellas se encuentra con lo que "hace que haya".

“(...), el hombre, al existir, no solo se encuentra con cosas que 'hay' y con las que tiene que hacerse, sino que se encuentra con que 'hay' que hacerse y 'ha' de estar haciéndose" (55).

En este sentido, Zubiri afirma que hay "algo" que fuerza nuestra propia realización personal. Pero ese algo no es una cosa, porque ninguna cosa determinada posee la fuerza para apoyar e impulsar la existencia humana completa.

(53) Lo interesante aquí es que Zubiri niega que se trate de "algo físico" pero en su filosofía madura lo físico indica precisamente lo real. En su planteamiento maduro afirmará que lo que hace ser al hombre es algo físico.

(54) NHD 428.

(55) NHD 428. 
$8^{\mathrm{a}}$ tesis:

Este hacer que haya existencia no se manifiesta al hombre como una simple obligación de ser, porque la obligación es siempre algo posterior al fenómeno radical del que aquí se trata. Es aquí cuando Zubiri introduce la "religación" como vínculo ontológico del ser humano, al afirmar que:

"La presunta obligación es consecuencia de algo más radical: estamos obligados a existir porque, previamente, estamos religados a lo que nos hace existir" (56).

La religación es una vinculación no consecutiva sino constitutiva de la misma existencia (57). La distinción entre la obligación y la religación radica en que mientras la primera indica el sometimiento de la existencia humana una vez que ya está constituida, la segunda apunta a una fuerza absolutamente distinta que apoya a tergo a la existencia, y tiene el carácter de una fuerza originaria de la que el hombre viene o procede. En la religación el hombre no está sometido, sino que, apoyado y religado, está doblegado reconociendo y acatando la fuerza que lo hace ser.

"En la religación estamos más que sometidos; porque nos hallamos vinculados a algo que no es extrínseco, sino que, previamente, nos hace ser. (...) En la religación, por el contrario, no 'vamos a', sino que previamente 'venimos de'. Es, si se quiere, un 'ir', pero un ir que consiste no en un 'cumplir', sino más bien en un acatar aquello de donde venimos, 'ser quien se es ya"' (58).

Para Zubiri, en la religación el hombre no está obligado sino apoyado y, por eso, la actitud humana que le corresponde es la de doblegarse ante la raíz que lo apoya y no la de someterse a esta (59).

$9^{\text {a }}$ tesis:

La religacion nos muestra y hace actual la "fundamentalidad" de la existencia humana. El fundamento es, a la vez, la raíz y el apoyo y, por tanto, no tiene un carácter primario de tipo conceptual sino de mayor radicalidad, es la causa de que "estemos" siendo. La religación es la ligadura al fundamento de la persona, es la ligadura a su apoyo y raíz.

$10^{\mathrm{a}}$ tesis:

La afirmación del existir como "existir con" (las cosas, los otros, nosotros mismos) requiere de la comprensión del "con" como aspecto que pertenece intrínsecamente al mismo ser del hombre. El "con" de la existencia nos indica que la religación religa en la existencia humana al mundo entero.

(56) NHD 428.

(57) Cf. SH 433. "El hombre está finalmente obligado a sí mismo, pero en su obligación no hace sino realizar una ligadura mucho más honda y radical, aquella que toca lo más radical y hondo de su personalidad, que es precisamente la religación de ultimidad".

(58) NHD 428-429.

(59) Cf. HD 94; PFHR 92. 
A propósito Zubiri señala que:

"La religación no es algo que afecte exclusivamente al hombre, a diferencia, y separadamente, de las demás cosas, sino a una con todas ellas. Por esto afecta a todo. Solo en el hombre se actualiza formalmente la religación; pero en esta actualidad formal de la existencia humana que es la religación aparece todo, incluso el universo material, como un campo iluminado por la luz de la fundamentalidad religante" (60).

En el hombre todas las cosas están religadas y, en consecuencia, todas ellas tienen el problema de la fundamentalidad última, el problema de la nihilidad radical, pero solo desde el hombre se ve que el universo entero está religado.

Esta amplia comprensión de la religación hace posible que el hombre pueda tener religión como una plasmación posible de la misma religación. La religación es la dimensión personal en la que se inscriben las religiones, en la que tienen su raíz y fundamento. Esta idea, que a juicio de Zubiri, está presente en la tradición teológica y filosófica, no está libre de dificultades debido a la imprecisión de algunos de sus conceptos. Si bien es claro que para el cristianismo el único capaz de la revelación es el hombre y en él el universo, la explicitación de este aspecto fundamental no ha estado libre de imprecisiones y errores que dificultan la comprensión de lo que se está señalando con la religación zubiriana. En función de esto, analiza brevemente la dificultad de entender la religación desde la perspectiva de la religio naturalis, de los escolásticos, o desde el concepto tradicional de principium originale de Buenaventura (61).

Ante esta situación, Zubiri insiste en la fundamentalidad de la religación ante toda posible ulterior configuración religiosa de la misma. Por tanto, la actitud religiosa no es ni un simple sentimiento, ni un conocimiento, ni un acto de obediencia, ni de incremento para la acción, sino la actualización del ser religado del hombre. En la religión de lo que se trata es de reconocer y acatar el fundamento para ser y ello es lo fundamental del culto.

\section{$11^{\text {a }}$ tesis:}

Finalmente, Zubiri reafirma que es en la apertura existencial en la que se muestra la religación. E indica que tal como el hombre descubre las cosas al estar abierto a ellas, en el estar religado descubre que hay lo que religa y constituye la raíz fundamental de su existencia. Pero aquí es preciso ser cautos, ya que lo que es patente al hombre en la religación no es directamente Dios sino la deidad. Es la razón la que debe precisar qué es la deidad, ya que por simple intuición el hombre no sabe que esta es Dios. En este sentido, la religación fuerza al hombre a poner en juego su razón para precisar y justificar la realidad de Dios, ya que en estricto rigor ella solo abre al ámbito de la deidad. Con esto queda claro que el planteamiento zubiriano no solo no niega la necesidad de la razón, sino que la exige y valora radicalmente.

(60) NHD 429.

(61) Cf. NHD 430-431.

(62) NHD 432. 
"La deidad se nos muestra como simple correlato de la religación; en la religación estamos "fundados", y la deidad es "lo fundante" en cuanto tal. Inclusive el intento de negar toda realidad a lo fundante (ateísmo) es metafísicamente imposible sin el ámbito de la deidad: el ateísmo es una posición ante la deidad" (62).

Si el hombre se encuentra constitutivamente vertido al problema del fundamento o deidad, su respuesta siempre deberá darse dentro de este ámbito. Y en este sentido, cualquier intento por demostrar la existencia de Dios o explicar sus atributos, supone previamente la religación a la deidad (63) y la primaria intelección del problema de Dios, ya que solo ulteriormente es posible cualquier demostración de su existencia o explicitación de sus atributos.

Cuando Zubiri afirma que el hombre está finalmente religado a Dios, se está refiriendo a Dios como el fundamento fundamentante que como apoyo hace ser al hombre.

“(...), así como la exterioridad de las cosas pertenece al ser mismo del hombre, en el sentido arriba indicado, esto es, sin que por esto las cosas formen parte de él, así también la fundamentalidad de Dios "pertenece" al ser del hombre, no porque Dios fundamentalmente forme parte de nuestro ser, sino porque constituye parte formal de él el "ser fundamentado", el ser religado. Dios no es nada subjetivo, como tampoco lo son las cosas externas. Existir es, en una de sus dimensiones, estar habiendo ya descubierto a Dios en nuestra religación" (64).

El hombre religado no se encuentra "con" Dios, como sí se encuentra con las cosas, sino que se encuentra "en" Dios. Por eso, en la religación no se trata de un ir "hacia", sino de un estar "viniendo" desde Dios, en un tener que "hacerse y hacer". En este sentido, todo posterior "ir hacia" Dios es un "ser llevado" por él mismo. Si en la apertura ante las cosas el hombre se encuentra con las cosas y se pone frente a ellas, en la apertura y encuentro con Dios, el hombre ya "está puesto" en la existencia, ya esta implantado en el ser, como viniendo "desde" Dios.

Por esto Zubiri insiste en que el hombre no puede ser entendido desde sí mismo, sino desde Otro. En esta perspectiva debe comprenderse su alusión al discurso de Pablo de Hchs 17, 28, que indica que: "nos movemos, vivimos y somos en Él". Entendiendo el sentido del "en" como el estar constitutivamente religado a Dios. El problema de Dios es finalmente para Zubiri el problema de la religación.

"Como dimensión ontológica, la religación patentiza la condición de un ente, el hombre, que no es ni puede ser entendido en su mismidad, sino desde fuera de sí mismo" (65).

(63) Cf. NHD 444.

(64) NHD 432.

(65) NHD 433. 
Concluye su análisis afirmando que el problema de Dios es una dimensión particular y constitutiva que le está planteada a todo hombre por el solo hecho de estar implantado en la existencia.

\subsection{Errores a evitar}

Luego de este análisis, es evidente que para Zubiri el problema de Dios afecta al ser mismo del hombre, porque este esta viniendo y siendo "en" Dios como su fundamento. Por eso, toda discusión sobre cuáles son las facultades que llevan al hombre a encontrarse con Dios resulta estéril, descartando explícitamente dos comprensiones erróneas sobre este asunto: en primer lugar, aclara que su visión no tiene relación con lo que se conoce como "filosofía de la acción" porque esta indicaría algo práctico, mientras que su propuesta apunta al ser del hombre y no a algo práctico, ni teórico, ni de pensamiento, ni de vida (66). Zubiri insistirá en que Dios es el fundamento de la existencia que se hace presente como verdadero problema en la constitutiva religación del ser del hombre; y, en segundo lugar, alerta contra una comprensión reduccionista de su planteamiento que restrinja el problema de Dios a una cuestión primariamente de mero conocimiento. El hombre es llevado a conocer por su propio ser, abierto y religado, es la propia existencia humana la que es un intento de conocimiento de las cosas y Dios. Reconociendo la existencia de un problema intelectual en torno a Dios, Zubiri niega radicalmente que este sea de carácter primario, ya que de lo que se trata en la religación es del ser del hombre y no solo de un problema de conocimiento.

"Dios no es algo que está en el hombre como una parte de él, ni es una cosa que le está añadida desde fuera, ni es un estado de conciencia, ni es un objeto. Lo que de Dios haya en el hombre es tan solo la religación en que somos abiertos a Él, y en esta religación se nos patentiza Dios" (67).

\subsection{Dios y el problema del ser}

Entre las distintas capacidades del hombre está obviamente la de conocer. El entendimiento conoce si algo es o no es, si es de una u otra manera y por qué es de esa manera y no de otra. Zubiri comprende que como el entendimiento se mueve siempre en el "es" se hace posible pensar erróneamente que esta es la forma primaria en que el hombre entra en contacto con las cosas.

Al conocer se entiende lo que "hay" como "siendo" (68). Efectivamente las cosas se convierten en entes, pero el "ser" supone siempre el "haber" (69). El ser es siempre ser de lo que hay, de un haber que se constituye en la radical apertura en que el hombre está abierto a las cosas y en que se encuentra con estas. Como este

(66) Dejando traslucir una aceptación y crítica del planteamiento de Blondel.

(67) NHD 435

(68) Cf. NHD 436.

(69) Cf. NHD 436-440. 
encontrarse pertenece a su ser, también le pertenece la intelección de las cosas, es decir, el entender lo que son. Como el hombre está abierto a las cosas, el ser que el entendimiento primariamente entiende es el ser de las cosas, hecho que la filosofía debe interpretar, porque no se trata de un simple transferir el ser de las cosas al existir humano, sino de una nueva ratio entes, a la que se llega a través de una dialéctica ontológica, entendida por Zubiri como algo que forma y es anterior al concepto (70).

En el caso específico del problema de Dios, el entendimiento nos indica que no solo hay cosas, sino también aquello que religa y fundamenta la existencia, es decir, Dios. Un "hay" distinto a las cosas porque su propio contenido es problemático. Con esto, el planteamiento zubiriano de la religación no elimina, ni hace superflua la intelección de Dios, sino que conduce expresamente a ella con su radical problematismo porque obliga a plantearse dicho problema.

"Pues si, en efecto, fue radical el retorno que nos llevó desde las cosas a entendernos a nosotros mismos, es todavía más radical aquel retorno en que, sin pararnos en nosotros mismos, somos llevados a entender, no lo que 'hay', sino lo que "hace que haya"' (71).

Zubiri es claro al reafirmar que Dios no es lo que "hay", sino lo que "hace que haya" algo. La existencia humana y Dios no son problemas distintos que se conecten por un puente. Dios es el fundamentar mismo, y desde un punto de vista humano, es el estar fundamentando, es decir, es la deidad. En este sentido, entiende la imposibilidad de identificar a Dios con el ser, porque Dios está allende el ser, en Él el haber rebasa infinitamente el ser. En este punto sigue la tradición de Eckhardt y la de la mística cristiana (72). Zubiri entiende que de lo que se trata es de descubrir una nueva ratio entis que vuelve todo problemático, por eso el problema que Dios plantea no se refiere a Él exclusiva e independientemente, sino que se refiere también a todo lo demás porque desde Él todo adquiere un sentido nuevo y distinto.

La existencia religada es una "visión" (73) de Dios en el mundo y del mundo en Dios. Una comprensión que lejos de ser una visión intuitiva, como pretendía el ontologismo, es la simple manifestación que acontece en la fundamentalidad religante que lo ilumina todo con una nueva ratio entis, que posibilita posteriormente un intento de demostración discursiva de la existencia y de los atributos entitativos y operativos de Dios. Solo cuando se intenta conceptualizar esta ratio entis y darle su justificación ontológica es cuando el hombre se ve forzado a intentar una demostración discursiva de la existencia y atributos entitativos de Dios. Pero es preciso indicar claramente que para Zubiri dicha demostración nunca es el descubrimiento primario de Dios.

(70) Cf. NHD 439, n. 1.

(71) NHD 440.

(72) Cf. NHD 441, n. 1.

(73) Cf. NHD 443. En este punto existe un importante desarrollo posterior en su pensamiento que llega a cuestionar la hegemonía que ha adquirido la visión como órgano privilegiado para el encuentro con Dios; IRE 99-113; HD 103-104, 108-109. 
Respecto de este punto indica:

"Pero esto será siempre una explicación ontológica, lograda dentro de una previa visión de las cosas: la visión que nos confiere esa primaria vinculación por la que todo se nos muestra religado a Dios" (74).

El análisis zubiriano no solo no elimina la marcha del entendimiento hacia Dios sino que la hace radicalmente necesaria. Sin entrar a prejuzgar sobre la índole propia de la divinidad, Zubiri logra aclarar la dimensión en que se encuentra planteado el problema de Dios, sosteniendo que se trata de la constitutiva religación de la existencia del hombre. E indica que:

"Desde el momento en que entender es siempre entender lo que hay, resultará que toda existencia tiene un problema teológico, y que, por tanto, es esencial a toda religión una teología" (75).

\subsection{Religación y libertad}

Ante la generalizada percepción de que un planteamiento como el de la religación o cualquier referencia a Dios, restringen la libertad humana, Zubiri realiza unas breves aclaraciones.

Comienza reconociendo la pluralidad de significaciones que tiene el concepto libertad, indicando que este puede ser entendido según su uso en la vida, es decir, como el acto libre o no libre; pero también en un sentido más hondo, como la liberación de la propia existencia.

Zubiri señala que en la religación el hombre no "tiene" primariamente libertad en ninguno de estos sentidos, porque tanto su "uso común" como la "liberación" emergen de la previa y radical constitución de un ente cuyo ser es "constitutivamente libertad". En la religación el hombre "está" siendo efectivamente persona libre.

"Como uso de la libertad, la libertad es algo interior de la vida; como liberación, es el acontecimiento radical de la vida, es el principio de la existencia, en el sentido de transcendencia y de vida; como constitución libre, la libertad es la implantación del hombre en el ser como persona, y se constituye allí donde se constituye la persona, en la religación" (76).

Solo la religación hace posible la libertad, porque la hace posible como libertad "para" y no solamente como libertad "de" (77), y ello porque la libertad solo existe en un ente implantado como máxima fundamentalidad de su ser. No hay libertad sin fundamento. En este sentido, Zubiri sostiene que Dios no es un límite

(74) NHD 444.

(75) NHD 445.

(76) NHD 446.

(77) Algo que posteriormente completará indicando que es fundamental la libertad "en", es decir, situada en la realidad. Cf. HD 328-330. 
extrínseco a la libertad humana, como se sostiene en algunas filosofías de su época, sino que, por el contrario, como fundamentalidad es lo que confiere al hombre su ser libre como uso de su libertad y liberación, porque lo constituye en ser fundamentado como quien existe y cuya existencia consiste esencialmente en ser libremente.

"Sin religación y sin religante, la libertad sería, para el hombre, su máxima impotencia y su radical desesperación. Con religación y con Dios, su libertad es su máxima potencia, tanta que con ella se constituye su persona propia, su propio ser íntimo e interior a él, frente a todo, inclusive frente a su propia vida" (78).

Por estar religado, el hombre como persona libre es un sujeto absoluto frente a lo demás e incluso, en cierto modo, frente a Dios mismo, porque estando religado está implantado en la existencia haciéndose, es decir, como constitutivamente suyo. En la religación el hombre cobra su libertad, su relativo ser absoluto, absoluto porque es "suyo" y relativo porque es "cobrado".

\section{BREVE CONCLUSIÓN}

Quisiera concluir, indicando tan solo tres ideas que me parecen fundamentales para una adecuada comprensión del planteamiento zubiriano inicial sobre el problema de Dios.

En primer lugar, es preciso no perder de vista que lo expuesto en este artículo pertenece a una época determinada del desarrollo zubiriano y su contenido debe ser comprendido en el contexto mayor de su pensamiento general. Pero a pesar de esto, es importante reconocer que la estructura fundamental con que intenta responder al Problema de Dios en 1936, enriquecida y mejor elaborada, sigue siendo válida en su etapa de madurez.

En segundo lugar, es importante reconocer que Zubiri logra abordar y reposesionar la cuestión de Dios en un nivel filosófico más adecuado a las necesidades de su tiempo, al afirmar que dicho problema es una dimensión particular y constitutiva de todo hombre por el solo hecho de estar implantado en la existencia. Zubiri se atreve a decir una palabra seria, clara y consistente, sobre un tema que es evitado por no pocos filósofos en la época y, en este sentido, realiza un verdadero esfuerzo no solo por reposicionar filosóficamente el tema de Dios, sino por centrar adecuadamente su problematicidad sin negar la necesidad y el valor del conocimiento humano.

$\mathrm{Y}$, en tercer lugar, siendo muy clara e importante la influencia heideggeriana en Zubiri, sobre todo entre los años 1930 y 1944, es preciso reconocer el notorio conflicto que comienza a producirse entre ambos pensamientos. Efectivamente, $\mathrm{Zu}$ biri discutirá inicialmente algunas tesis fundamentales de Ser y tiempo, llegando a cuestionar, en sus obras de madurez, las ideas heideggerianas de la existencia, el sentido, la comprensión, la verdad como desvelación, el ser y la historicidad.

(78) NHD 446. 
Un progresivo distanciamiento que se vislumbra germinalmente en el texto zubiriano que hemos revisado y que da cuenta de algunas líneas fundamentales de un planteamiento distinto, y filosóficamente fundamentado, que todavía espera ser teológicamente comprendido, asumido y superado.

\section{RESUMEN}

Este estudio intenta mostrar el planteamiento inicial de Xavier Zubiri sobre la cuestión de Dios presente en su artículo titulado "En torno al problema de Dios", del año 1936, explicitando la influencia del pensamiento heideggeriano y los indicios de un camino propio, particular y específico, que ya comienza a vislumbrarse.

Después de unos breves datos biográficos se repasan algunos elementos que permiten comprender la continuidad y distinción entre ambos planteamientos filosóficos.

En el cuerpo del texto, se da cuenta del intento zubiriano por explicitar el "supuesto" filosófico que estaría a la base de gran parte de las demostraciones o negaciones contemporáneas de la existencia de Dios, que lo consideran un hecho externo y añadido al hombre. Ante este panorama, es muy claro el esfuerzo zubiriano por mostrar la cuestión de Dios como un verdadero problema que afecta a todo el hombre y a todos los hombres, por ser una dimensión constitutiva y radicalmente propia de la existencia humana. Una propuesta en la que se reafirma la importancia de la razón humana pero se niega el que se trate de un problema primario de orden meramente epistemológico. Zubiri insiste en que Dios es un verdadero problema para el hombre por el mero hecho de ser hombre, por estar religado a la deidad o fuerza de lo real que realmente lo hace libre.

Palabras clave: Dios: Fundamento, fundamentante. Persona: Constitutiva indigencia. Existencia dada, implantada. Religación (a tergo). Paradoja: Dios lo más propio y lo más otro.

\section{ABSTRACT}

This study intends to show Xavier Zubiri's initial position on the question of God found in his article titled, "Regarding the Problem of God", from 1936, specifying both the influence of Heideggerian thought and the traces of his own, particular and specific path that here begins to visibly emerge. After a few brief biographical facts, some elements that allow us to understand the continuity and the distinction between both philosophical approaches will be reviewed. In the body of the text, account is given of the Zubirian attempt to specify the philosophical "supposition" that would be the basis for a great number of contemporary demonstrations or refutations of the existence of God, which consider God an external fact, added on to humanity. In the face of this panorama, the Zubirian effort clearly shows the question of God as a true problem, affecting the whole human person and all of humanity, due to its being a dimension both constitutive and radically characteristic of human existence. This is a proposal in which the importance of human reason is reasserted, while at the same time denying that a primary problem of a merely epistemological order is the issue. Zubiri insists that God is a true problem for the human being, by the mere fact of being human, by being strongly attached to the deity or force of the real, which truly makes him free.

Key words: God: Basis - Foundation, Person: Constitutive poverty, Given, implanted existence, Religation (from behind), Paradox: God as maximally Self and as maximally Other. 